\title{
Microbes or "Macrobes" and how should they be Dealt with?
}

\section{Oto Melter}

Department of Medical Microbiology, Charles University, Czech Republic

Free-living, commensal or pathogenic microbes prefer to live as "macrobes" if they can. The simplest example of such a "macrobe" is a microbial colony growing in vitro. Even if the "macro organism" is an artificial organism it could probably behave analogically to a natural one such as biofilm or other microbial communities still not yet specified and characterized adequately. Microbial cells within such a "macrobe" are diversified and hence specialized to fulfil various functions essential to their adhesion, metabolism and propagation, which will ensure survival of such a microbial organism in competitive environment.

Heterogeneity of such a microbial population is determined by genetic, epigenetic or unknown features which we should just study and interpret with caution in the future. Such a microbial population cultured from a sample can be called an isolate and together with other such populations constitute a microbial strain. Thus, vital features ensuring phenetic heterogeneity are prerequisites for successful evolution of any microbe. Only a few facts involved in life of such an artificial microbial colony are specified further. Microbial cells having contact with a solid culture medium tend to swarm or to glide along the agar surface to take a free space and potential new source of nutrients. Other adjacent cells are specialized to produce higher concentration of metabolic enzymes in order to utilize the newly acquired nutrients. So the population can consist of a lot of diversified populations.

All the microbial behaviour mentioned above is feasible only thanks to active communication among microbial cells but also subpopulations within such a population and species or within a microbial world as well. The communicating microbial cells can sense density of their cells (quorum sensing) by using small hormone-like molecules or detecting a rate of increased concentration of a released diffusible substance (diffusion sensing), which enables them to sense a free environment as a potential source of essential nutrients. Efficiency sensing combining both of the communication skills described above is suggested to take place in most of microbial social activities.

An example of a biofilm which can measure up to few square meters or a patient's lower respiratory tract afflicted by a chronic bacterial infection support the theory of microbial "macroorganism". Bacteria that attach to surfaces aggregate in a hydrated polymeric matrix of their own synthesis to form biofilms. Formation of sessile communities resistant to antimicrobials is significant for treatment of chronic patients especially if they can give rise to non-sessile cells-planktonic bacteria that can rapidly multiply and disperse. Studies of biofilms have revealed differentiated and structured groups of bacterial cells with the community properties.

Epidemics caused by successful epidemic strains described in many bacterial species could also be an argument for successful microbial adaptive radiation. Furthermore, if we stress attention only to cells within a bacterial strain population we could also realize the cell diversity including adhesion properties, antibiotic susceptibility, virulent factors and persistence. Small colony variants (SCV) of Staphylococcus aureus in chronic patients could be mentioned as a fitting example. There is a subpopulation of staphylococcal cells within a wild type strain of $S$. aureus which evolve because of appearance of spontaneously mutated cells or cells regulated by metabolic regulator - npcRNA (non-protein coding RNA) start to prevail in the new population. The subpopulation is metabolically defective but because of different structure and minimized production of a crucial virulent factor (e.g. alpha toxin), it is more resistant to antibiotics and of low virulence. Hence, they cannot primarily destroy host cells and they persist intracellularly for months not influenced by any antibiotics and avoiding the host immune system. When antibiotic therapy eliminating the extracellular wild type population finishes, they usually revert to the wild type population, which disrupts invaded host cells, and the infection progresses and exacerbates.

Another interesting example of adaptive microbial evolution or radiation is a phenetically diverse population of Pseudomonas aeruginosa in cystic fibrosis patients. Many morph types characterized by different appearance, size and pigmentation of bacterial colonies can be cultured from one single sample from such chronically infected patients. Susceptibility to antibiotics or other biological properties of such morph types can also differ substantially. Continually changed conditions within the patient respiratory tract damaged by the chronic inflammation have a main impact on constant diversification of the population and its survival in the niche. Higher mutation rate determined by a defective DNA repair system is most likely responsible for such curious phenomena.

Antigenic variations within bacterial populations were also described by many microbiologists. However, some of the variations were detected just as a secondary outcome in the course of clonal analysis or analysis of surface structures. It is suspected that essential role of such variation or diversification is most likely an immunological mimicry of the agent to evade host innate or specific immunity.

Thanks to an enormous number of microbial cells within such a "macrobe", microbial evolution is likely to resemble adaptive radiation in higher organisms, which occurs through continual changes rather than changes in saltations. Higher organisms evolve in process of sympatric or allopatric speciation when two thriving species evolve from the same ancestor in the same or different geographic area, respectively. Commensal or pathogenic microorganism evolution could be specified as the sympatric one because of usually enormously high density of microbial cells exist within a hosts what determines common direct or indirect contacts of microbial species between each other within one or new hosts. Bartonella henselae made its host specificity narrower to shorten its genome substantially and hence creating a new human pathogen - B. quintana, which is another nice illustration of a newly evolved species.

*Corresponding author: Dr. Oto Melter, Department of Medical Microbiology, Charles University, Czech Republic, E-mail: oto.melter@lfmotol.cuni.cz

Received December 21, 2012; Accepted December 26, 2012; Published December 29, 2012

Citation: Melter O (2013) Microbes or "Macrobes" and how should they be Dealt with? Clin Microbial 1: e103. doi:10.4172/2327-5073.1000e103

Copyright: @ 2013 Melter O. This is an open-access article distributed under the terms of the Creative Commons Attribution License, which permits unrestricted use, distribution, and reproduction in any medium, provided the original author and source are credited. 
Even if microorganisms do not reproduce sexually by choosing various alleles from parent cells, they can acquire an enormously large pool of various genes by horizontal gene transfer or other mechanisms such as reversible large-scale genomic inversion called also chromosomal flip-flop.

Microbial "macrobes" differ substantially from higher organisms' sensu stricto in their structure. As their bodies are not compartmentalized like in higher organisms and actually they are studied with using a microscope hence they are named as microbes. A microbial world started four milliard years ago and thus it is much more ancient than that of dinosaurs and ours. And microbes have never stopped learning and evolving and they are still evolving! We can call them unicellular or primitive organisms but because they passed such a long historical way they are experienced and able to use many sophisticated strategies. Thus, we can never win the battle against them, but with a humble understanding of their history, we could increase our efficiency in infectious disease treatment. 